tric "fistula", not "ulcer", to investigate gastric function, and so forth. There are also omissions, possibly the most obvious being that of Galen, the greatest medical man before the 17th century and a military surgeon. These may seem trivial defects and individually they mostly are. They are, however, useful diagnostic signs for the ovaluation of the book's contents. For those in a position to assess this material critically there is much useful information presented in a clear and attractive style.

Edwin Clarke

\section{THREE DIMENSIONAL BIOLOGY}

\section{Molecular Asymmetry in Biology}

Vol. 1. By Romald Bentley. (Molecular Biology: an International Series of Monographs and Textbooks.) Pp. xi+322. (Academic Press: New York and London, December 1969.) 131s.

THIs book, which is the first of two volumes, provides a working background of stereochemical principles and nomenclature, and discusses some general aspects of stereochemistry in biology. It might indeod have been entitled "Stereochemistry for Biologists". It is an excellent and detailed survey for this purpose, and will also serve as a reference book for many organic chemistis. The second volume, which is to follow, will discuss some enzymatic reactions for which detailed stereochemical information is available, and will review the absolute configurations of biologically significant compounds.

The first chapter, dealing with stereochemical principles, discusses the various types of isomerism, both those which are well treated in classical textbooks and more recent ones such as cyclo-stereo-enantionerism. The second chapter deals at length with nomenclature, which in this context is far from being an arid and formal subject. The development of a universal system of stereochemical nomenclature by Cahn, Ingold and Prelog during a period of nearly twenty years has led to much fundamental thinking, which might not have been stimulated otherwise. The author provides as simple an introduction to the application of the sequence rule as can reasonably be expected. Many workers find the application of this rule difficult, but there is no excuse for not "following the drill" when it is set out as Bentley has done here. $\AA$ future edition of this book will need to spend much time on the analysis of all types of stereoisomerism in terms of "simplexes", as sketched by Prelog in his Robert Robinson Lecture to the Chemical Society in Dublin in 1968 and dealt with more fully in a lecture in Hamburg in 1969 (as yet unpublished).

Chapter three begins with some very simple concepts regarding reaction mechanisms, including thoso of kinctic and thermodynamic control, and definitions of the terms "stereoselective" and "stereospecific". The various methods for the preparation and resolution of optically active compounds and the different types of asymmetric synthesis are discussed in detail with good examples. Here, as elsewhere, a wealth of good formulae provide a vital contribution to the book's value.

Chapter four deals with the important concept of pro-chirality. This term implies a situation in which a tetrahedral carbon atom is attached to two identical groups and to two other different groups, for example, Caabc. For most chemical and physical purposes, the two groups " $a$ " are indistinguishable. In any reaction with another molecule, which is itself chiral, however, these two groups are different. This concept was first evolved by Ogston in relation to enzymatic reactions in 1948, and it has been generalized and further developed, largely by Hirschmann and by Hanson, in subsequent years.

It is an interesting sidelight on the way in which many scientific ideas develop; here we have a concept which is really implicit in tho idca of the tetrahedral carbon atom and the chirality deriving from it, but which lay completely unnoticed for 70 years until application in another field of science, onzymology, brought it to the fore. Even then some further years elapsed before the generality of this idea was recognized. In this book the conditions in which pro-chiral groups can be differentiated, and the terminology used to describe them, are discussed in detail.

Chapter five discussos isotopic substitution and chirality. Many of the most elegant studies on pro-chirality have depended upon the use of isotopes, by which a pro-chiral situation is turned into a chiral situation, which can then be explored by a variety of physical techniques, particularly those of optical rotation. Key compounds which are discussed (with an extensive series of diagrams) include ethanol, malic acid, succinic acid and malonic acid, all labelled with deuterium.

The final chapter deals with some aspects of the biological significance of stereoisomers. Here the wealth of material is such that the author chooses particular examples for discussion. The topics considered are the natural occurrence of compounds in both enantiomeric forms; the biological role of D-amino-acids; the stereochemical approach to structure-activity relationships; the utilization of enantiomers by plants and microorganisms; the activity of enzymes with both enantiomers as a substrate; and general physiological responses to stereoisomors.

This volume is not very expensive, although it will hardly have as wide a circulation in undorgraduate classes as one would like to see. Looked at in another way, if one takes Dr Bentley's excellent text for granted, a total of more than 850 structural formulae and nearly 600 references works out at a little more than $1 d$ per formula or reference. I look forward to the appcarance of the second volume of this work, and organic chemists may expect more and better questions from their biological colleagues as these books become widely known.

W. KLYNE:

\section{ELECTROPHYSIOLOGICAL METHODS}

Electrophysiological Analysis of Synaptic Transmission By J. I. Hubbard, R. Llinas and D. M. J. Quastel. (Monographs of the Physiological Society, Vol. 19.) Pp. ix + 372. (Arnold: London, December 1969.) $105 s$.

THe virtue of the monographs of the Physiological Society is that they are written by physiologists for physiologists. In this case the authors have set out to provide experimental physiologists and pharmacologists with the electrophysiological context in which details of synaptic mechanisms can be analysed. They havo not writton, indeed they did not intend to write, a textbook on synaptic transmission.

The first third of the book describes the electrochemis. try and electrical theory which are the foundation of electrophysiology. A lot of material is presented here and to cover it in one hundred pages nocessarily involves selection. More thought might have been given to the presentation of several topics. The section on cable theory and the consequences of cellular geometry is inadequate as a basis for the discussion of Rall's neurone model and Falk and Fatt's treatment of the complex membranous systems of muscle fibres. Without considerable previous knowledge a reader could be forgiven for getting lost in the section on this important but difficult subject. Chapter three contains sensible comments about electrophysio. logical methods; in particular, it points out some of the pitfalls associated with the Wheatstone bridge method for using the same intracellular microelectrode to pass current and measure potential.

Chapter four deals with the investigation of presynaptic function and gives a critical account of methods for estimating the quantal content of endplate potentials. 